\title{
Saccharomyces cerevisiae Oxidative Response Evaluation by Cyclic Voltammetry and Gas Chromatography-Mass Spectrometry
}

\author{
Cristiana C. Castro, ${ }^{\dagger}$ Caitriona Gunning, ${ }^{\ddagger}$ Carla M. Oliveira, ${ }^{\ddagger}{ }^{8}$ José A. Couto, ${ }^{\ddagger}$ José A. Teixeira, ${ }^{\dagger}$
} Rui C. Martins, " and António C. Silva Ferreira* ${ }^{* \ddagger}, \perp$

\author{
${ }^{\dagger}$ IBB-Institute for Biotechnology and Bioengineering, Centre of Biological Engineering, Universidade do Minho, Campus de Gualtar, \\ 4710-057 Braga, Portugal \\ ${ }^{\ddagger}$ Escola Superior de Biotecnologia-CBQF, Universidade Católica Portuguesa, Rua Dr. António Bernardino de Almeida, $4200-072$ \\ Porto, Portugal \\ ${ }^{\S}$ Departamento de Química \& QOPNA, Universidade de Aveiro, 3810-193 Aveiro, Portugal \\ "BioInformatics-Molecular and Environmental Biology Centre, Universidade do Minho, Campus de Gualtar, $4710-057$ Braga, \\ Portugal \\ ${ }^{\perp}$ Stellenbosch University, Private Bag X1, Matieland, 7602, Stellenbosch, South Africa
}

\begin{abstract}
This study is focused on the evaluation of the impact of Saccharomyces cerevisiae metabolism in the profile of compounds with antioxidant capacity in a synthetic wine during fermentation. A bioanalytical pipeline, which allows for biological systems fingerprinting and sample classification by combining electrochemical features with biochemical background, is proposed. To achieve this objective, alcoholic fermentations of a minimal medium supplemented with phenolic acids were evaluated daily during 11 days, for electrochemical profile, phenolic acids, and the volatile fermentation fraction, using cyclic voltametry, high-performance liquid chromatography-diode array detection, and headspace/solid-phase microextraction/gas chromatography-mass spectrometry (target and nontarget approaches), respectively. It was found that acetic acid, 2phenylethanol, and isoamyl acetate are compounds with a significative contribution for samples metabolic variability, and the electrochemical features demonstrated redox-potential changes throughout the alcoholic fermentations, showing at the end a similar pattern to normal wines. Moreover, S. cerevisiae had the capacity of producing chlorogenic acid in the supplemented medium fermentation from simple precursors present in the minimal medium.
\end{abstract}

KEYWORDS: bioanalytical methods, electrochemical sensors, mass spectrometry, chemometrics

\section{INTRODUCTION}

Fermentation is a long-used method of preservation. The primary fermentation that grape juice undergoes is alcoholic fermentation performed by wine yeasts, where glucose and fructose are converted to ethanol and carbon dioxide. ${ }^{1}$ In this way, a product with a high ethanol content immediately narrows the window for spoilage by other microorganisms, as does the lower $\mathrm{pH}$ and changes in the redox potential. ${ }^{2}$ Thus, alcoholic fermentation contributes to the resistance of the beverage to microbial spoilage. ${ }^{3}$ The challenges that oxidation poses to wine makers, however, are less easy to overcome. The wine's resistance to oxidation depends on its composition, its exposure to oxygen, and its antioxidants concentration. ${ }^{4}$ It is believed that the wine antioxidant capacity is related to the presence of antioxidants such as phenolic compounds. ${ }^{4}$ These compounds are currently of great interest in the wine industry, as a result of their purported health benefits and radicalscavenging properties.

To understand fermentation in detail, it is necessary to take a step back, to observe the overall process as a larger picture. Here, several methods can be used to monitor the "ongoing" fermentation. Electrochemistry and mass spectrometry methods can be used to understand yeast behavior under oxidative conditions and during the fermentation time course. Combining the information obtained from these detectors seems to be an advantage as it will be possible to correlate metabolic, chemical, and electrochemical responses providing tools to better understand the complexity of the overall system.

Cyclic voltammetry can be used for electroactive activity scanning. A voltammogram provides information about the type of antioxidants present, as well as quantitative information about the likelihood of oxidation of particular substances, ${ }^{6}$ enabling the understanding of how these compounds are metabolized and changed during fermentation.

Key volatile metabolites can be identified and quantified using a headspace/solid-phase-microextraction/gas chromatography-mass spectrometry (HS-SPME/GC-MS) technique by directly integrating chromatogram peak areas (namely target mode) or by a nontargeted methodology, an unbiased approach, toward understanding the overall biological system. ${ }^{7,8}$ The nontargeted approach allows a faster metabolic overview, avoiding the time-consuming need for any prior assignment of chemical classification of the molecular structure for hundreds of data sets. ${ }^{9}$

Received: March 8, 2012

Revised: June 25, 2012

Accepted: July 2, 2012

Published: July 2, 2012 


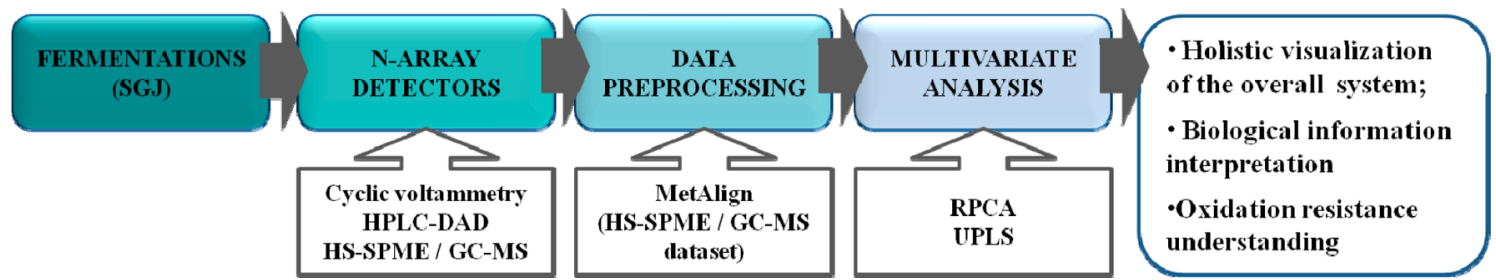

Figure 1. Schematic representation of the pipeline presented in the manuscript.

Studying Saccharomyces cerevisiae metabolism during fermentation is effectively a process of taking a series of snapshots of the metabolism at different stages of a very dynamic process, ${ }^{10}$ where some fermentation pathways are being activated, while others are being down-regulated.

In this study, the question of how yeast metabolism affects the concentration of phenolic compounds was addressed and monitored indirectly and directly, using cyclic voltammetry and HPLC/DAD/MS detection, respectively. In addition, the effect of the phenolic acids on the aromatic profile of the beverage was explored using HS-SPME/GC-MS analysis (Figure 1), conducted using both nontarget and target approaches. The information gathered by the different detectors used was then categorized using statistical methods and structured in terms of similarities and differences between samples, allowing the biological information interpretation and the oxidation resistance interpretation.

\section{MATERIALS AND METHODS}

Minimal Medium Preparation Protocol. Figure 2 presents the minimal medium preparation protocol for all fermentations studied.

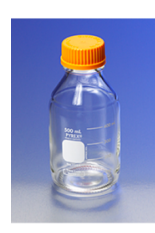

i) Control

$\mathrm{V}=\mathbf{9 0 0} \mathrm{ml}$
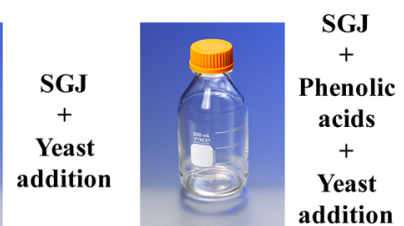

ii) Replica 1 $\mathrm{V}=900 \mathrm{ml}$

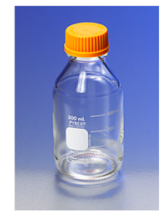

iii) Replica 2 $\mathrm{V}=900 \mathrm{ml}$
Figure 2. Methodology used for fermentation media preparation: SGJ, synthetic grape juice; Control, fermentation in the absence of phenolic acids; and Replica 1 and Replica 2, fermentations with phenolic acids supplemented medium (two replicates).

For this purpose, $3 \mathrm{~L}$ of a synthetic grape juice (SGJ, in section 2.2) was prepared and adjusted as follows: (i) addition of yeast (control) and (ii and iii) addition of yeast and phenolic acids (replica 1 and replica 2).

SGJ. A SGJ, a less complex and more reproducible matrix, was made according to the procedure described by Ciani and Ferraro. ${ }^{11}$ SGJ was produced by combination of three aqueous solutions (A, B, and C), prepared and sterilized separately. Solution A: $110 \mathrm{~g} / \mathrm{L} \mathrm{D-}$ glucose (99.5\%, Sigma), $100 \mathrm{~g} / \mathrm{L}$ D-fructose (99.0\%, Sigma), $10 \mathrm{mg} / \mathrm{L}$ ergosterol (95\%, Sigma-Aldrich), and $0.1 \%(\mathrm{v} / \mathrm{v})$ Tween 80 (for synthesis, Merck); solution B: $6.0 \mathrm{~g} / \mathrm{L} \mathrm{L}-(+)$-tartaric acid $(99.5 \%$, Merck), $3.0 \mathrm{~g} / \mathrm{L} \mathrm{L}-(-)$-malic acid (99.5\%, Fluka), and $0.5 \mathrm{~g} / \mathrm{L}$ citric acid (99.5\%, Sigma-Aldrich); solution C: $1.7 \mathrm{~g} / \mathrm{L}$ yeast nitrogen base (Bacto Difco), $2.0 \mathrm{~g} / \mathrm{L}$ casamino acids (Bacto Difco), $0.2 \mathrm{~g} / \mathrm{L}$ anhydrous calcium chloride (99.5\%, Merck), $0.8 \mathrm{~g} / \mathrm{L}$ L-arginine hydrochloride (98\%, Sigma), $1.0 \mathrm{~g} / \mathrm{L}$ L-(-)-proline (99\%, Sigma), 0.1 g/L L-(-)-tryptophan (98\%, Sigma), $0.1 \mathrm{~g} / \mathrm{L}$ phenylalanine (98\%, Sigma), and $0.1 \mathrm{~g} / \mathrm{L}$ L-tyrosine (98\%, Sigma).
Before they were sterilized, solutions $\mathrm{B}$ and $\mathrm{C}$ were adjusted to $\mathrm{pH}$ 3.5 with $\mathrm{NaOH}(2 \mathrm{M})$ and $\mathrm{HCl}(1 \mathrm{M})$. SGJ was then added to control (Figure 2i), replica 1 (Figure 2ii), and replica 2 (Figure 2iii).

Phenolic Acids Addition. The following phenolic acids were added to replica 1 and replica 2 (Figure 2) before the addition of the yeast: hydroxybenzoic acids-gallic acid monohydrate (99\%, SigmaAldrich), protocatechuic acid (99\%, Sigma- Aldrich), and vanillic acid (97\%, Sigma-Aldrich) — and hydroxycinnamic acids-caffeic acid (99\%, Sigma-Aldrich), para-coumaric acid (98\%, Sigma-Aldrich), and ferulic acid (99\%, Sigma-Aldrich). The final concentration of each phenolic acid was $15 \mathrm{mg} / \mathrm{L}$ each.

Yeast Addition. S. cerevisiae PYCC 4653 strain (Portuguese Yeast Culture Collection) was used for alcoholic fermentation in the control, replica 1, and replica 2 (Figure 2). Yeast cultures were previously grown in yeast extract-malt extract (YM) medium for a minimum of 2 days at $30{ }^{\circ} \mathrm{C}$ in an orbital incubator, then collected after centrifugation $\left(9000 \mathrm{rpm}, 15 \mathrm{~min}, 25^{\circ} \mathrm{C}\right.$ ), and resuspended in Ringer solution before addition to the fermentation medium.

The yeasts cells were pitched at about $1.0 \times 10^{6}$ colony-forming unit $(\mathrm{CFU}) / \mathrm{mL}$ into the culture medium and adjusted by microscopic enumeration with a cell-counting hematocytometer (Neubauer chamber; Merck) to start the fermentation. Fermentations of control and supplemented medium were carried out at $18{ }^{\circ} \mathrm{C}$ in $1 \mathrm{~L}$ sterile Schott flasks equipped with cotton plugs after filling with $900 \mathrm{~mL}$ of fermentation medium (Figure 2). The low temperature was chosen to simulate the white wine fermentation. ${ }^{12}$ Alcoholic fermentations were monitored for 11 days, until the viable counts of yeast cells had fallen below $1.0 \times 10^{6} \mathrm{CFU} / \mathrm{mL}$.

Samples were taken and analyzed daily, for 11 days, by HS-SPME/ GC-MS for metabolic changes, by cyclic voltammetry for electrochemical changes, and by HPLC-DAD for quantification of specific antioxidants. Residual sugars and ethanol were analyzed by HPLC-RI and used as fermentation control parameters.

Cyclic Voltammetry Analysis. Experiments were performed using a potentiostat (microAutolab Type III with an Autolab Faraday Cage), and voltammograms were obtained with a scan rate of $100 \mathrm{mV}$ with a set potential of $2.4 \mathrm{mV}$, between -0.2 and $1.2 \mathrm{~V}$. The working electrode was a $3 \mathrm{~mm}$ Glassy Carbon disk in combination with a Metrohm tipholder, cleaned by polishing with $3 \mu \mathrm{m}$ alumina powder between acquisitions. A saturated calomel electrode was used as a reference electrode in conjunction with a platinum counter electrode. Each acquisition required $12 \mathrm{~mL}$ of undiluted sample. Voltammograms were treated by General Purpose Electrochemical System (GPES) 4.9 software.

HPLC-DAD Analysis-Quantification of Phenolic Acids and para-Chlorogenic Acid. A Beckman model 126 quaternary solvent pump system, equipped with System 32 Karat 5.0 software and a 168 rapid scanning, UV-visible photodiode array detector, was used. The absorption spectra were recorded between 270 and $550 \mathrm{~nm}$. Stationary phase: Chromolith Performance RP-18e $(100 \mathrm{~mm} \times 4.6 \mathrm{~mm})($ Merck, Germany). Mobile phase: Solvent A: acetonitrile/water (5:95 v/v) (Merck pure grade and pure water) with 0.2\% TFA (Sigma-Aldrich, Germany). Solvent B: acetonitrile (100\%) (Merck pure grade) with $0.2 \%$ of TFA; flow rate $=3 \mathrm{~mL} / \mathrm{min}$. Employed was the following gradient: $0-2 \min (0 \% \mathrm{~B}), 2-6 \min (10 \% \mathrm{~B}), 6-10 \min (20 \% \mathrm{~B})$, $10-12 \mathrm{~min}(0 \% \mathrm{~B})$, post time of $3 \mathrm{~min}$. Each run took $15 \mathrm{~min}$ to complete, and all relevant compounds had eluted by $8 \mathrm{~min}$. Hydroxybenzoic and hydroxycinnamic acids were detected at 280 and $320 \mathrm{~nm}$, respectively. Along with these six phenolic acids (added 
to the medium preparation protocol), para-chlorogenic acid was also detected. Retention times were as follows: gallic acid, $0.8 \mathrm{~min}$; protocatechuic acid, $1.2 \mathrm{~min}$; vanillic acid, $3.0 \mathrm{~min}$; caffeic acid, 3.5 min; para-coumaric acid, $5.3 \mathrm{~min}$; and ferulic acid, $6.2 \mathrm{~min}$. Identification: Phenolics were identified by comparison with pure, authentic, commercially available standards' retention times and UVvisible photodiode array spectra.

HPLC-RI Analysis-Quantification of Residual Sugars and Ethanol. A Beckman model 126 quaternary solvent pump system equipped with an autosampler and a RI detector was employed. The acquisition was done using 32 Karat 5.0 software. Stationary-phase: Aminex hpx-87H $(300 \mathrm{~mm} \times 7.8 \mathrm{~mm})$ from Bio-Rad. Mobile-phase: sulfuric acid $(2.5 \mathrm{mM})$; flow rate $=0.6 \mathrm{~mL} / \mathrm{min}$.

LC-MS Analysis-Detection of Chlorogenic Acid. The chromatographic system consisted of a Prostar 210 LC pump (Varian, CA) coupled with a Varian 1200 triple quadrupole mass spectrometer (Varian) with electrospray ionization. A $5 \mu \mathrm{m} \mathrm{C18} \mathrm{column}(4.6 \mathrm{~mm} \times$ $100 \mathrm{~mm}$, Merck) was used for the separation at a flow rate of $0.4 \mathrm{~mL} /$ min. The separation was performed by gradient elution (eluent $\mathrm{A}$, water with $0.1 \%$ formic acid; eluent B, $100 \%$ methanol) in $33 \mathrm{~min}$. For MS/MS fragmentation, argon atoms were used (pressure, 1.20 mtorr; collision energy, $15 \mathrm{~V}$ ). Data were acquired by Varian LC-MS $1200 \mathrm{~L}$ Workstation. LC and MS-MS were established by ESI-LC/MS under negative ion mode. Structural identification of chlorogenic acid (MW $=354 ;[\mathrm{M}-\mathrm{H}]=353(191) ; \mathrm{RT}=15.82 \mathrm{~min}]$ was performed by comparison of the retention time and mass spectra of the phenolic standard with the supplemented medium. ${ }^{13}$

Gas Chromatography Analysis. HS-SPME. Volatile compound analyses using the analytical SPME technique were performed according to Ferreira and Guedes. ${ }^{14}$ The used fiber was coated with a divinylbenzene/carboxen/polydimethylsiloxane (DVB/CAR/ PDMS), 50/30 $\mu \mathrm{m}$ (Supelco, Bellefonte, PA). For each SPME analysis, $5 \mathrm{~mL}$ of sample was placed in a vial with $20 \mathrm{~mL}$ capacity together with a small stirring magnet, stirred at $1300 \mathrm{rpm}$, and spiked with an internal standard (IS) $(20 \mu \mathrm{L}$ of methanolic solution of 3octanol, $46.3 \mathrm{mg} / \mathrm{L}$ ) while immersed in a water bath at $36{ }^{\circ} \mathrm{C}$. The SPME needle then pierced the septum, and the fiber was extended through the needle to expose the stationary phase with the headspace of the sample for $5 \mathrm{~min}$. Afterward, it was removed from the vial and inserted into the injection port of the gas chromatograph for $5 \mathrm{~min}$. The extracted chemicals were thermally desorbed, at $220{ }^{\circ} \mathrm{C}$, and transferred directly to the analytical column. Fibers were cleaned before each microextraction process to prevent contamination by inserting the fiber in the auxiliary injection port at $220{ }^{\circ} \mathrm{C}$ for $30 \mathrm{~min}$.

Mass Spectrometry Analysis. Samples were analyzed using a Varian CP-3800 gas chromatograph (Walnut Creek, CA) equipped with a Varian Saturn 2000 mass selective detector and Saturn GC/MS workstation software version 5.51.14. The column used was a STABILWAX-DA $(60 \mathrm{~m} \times 0.25 \mathrm{~mm}, 0.25 \mu \mathrm{m})$ fused silica (Restek, Bellefonte, PA). The injector port was heated to $220^{\circ} \mathrm{C}$. The oven temperature was $40{ }^{\circ} \mathrm{C}$ (for $1 \mathrm{~min}$ ), then increased at $2{ }^{\circ} \mathrm{C} / \mathrm{min}$ to 220 ${ }^{\circ} \mathrm{C}$. The carrier gas was helium (Gasin, Portugal) at $1 \mathrm{~mL} / \mathrm{min}$ at constant flow. All mass spectra were acquired in the electron impact (EI) mode with the Ion Trap detector set as follows: transfer line, manifold, and trap temperatures 170,60 , and $150^{\circ} \mathrm{C}$, respectively. The mass range was $33-350 \mathrm{~m} / z$, with a scan rate of $6 \mathrm{scan} / \mathrm{s}$ and without solvent delay. The emission current was $50 \mu \mathrm{A}$, and the electron multiplier was adjusted according to the autotune procedure. The maximum ionization time was $25.000 \mu$ s with an ionization storage level of $35 \mathrm{~m} / z$ with a prescan time of $100 \mu \mathrm{s}$. The analysis was performed in full scan mode.

Two different approaches were used to analyze mass chromatograms: nontarget and target approaches. In the nontarget approach, the raw chromatograms were imported to MetAlign ${ }^{13}$ for spectral alignment and differentiation. The optimization of the software parameters was performed taking into account the need to preserve the original compounds features after preprocessing. The settings used in the preprocessing software are presented in Table 1. At the end of the alignment of data set, the Excel compatible output matrix was obtained and subjected to multivariate analysis techniques, namely,
Table 1. MetAlign Preprocessing Optimized Parameters

\begin{tabular}{lll}
\multicolumn{1}{c}{ MetAlign part } & \multicolumn{1}{c}{ parameter } & \multicolumn{1}{c}{ value } \\
& retention begin (Scan.nr) & 262 \\
& retention end (Scan.nr) & 4347 \\
& maximum amplitude & 10000 \\
A: baseline and noise & peak slope factor (xNoise) & 1 \\
elimination parameters & peak threshold factor (xNoise) & 2 \\
& peak threshold (Abs.Value) & 10 \\
& average peak width & 10 \\
& begin first region (Scan.Nr/ & $261 / 20$ \\
& Max.Shift) & $4347 / 20$ \\
& end first region (Scan.Nr/ & Max.Shift) \\
& prealign processing & iterative \\
Baximum shift per 100 scans & 35 \\
sets & min factor (xNoise) (first & $3 / 2$ \\
& iteration/last iteration): & \\
& min number of masses (first & $8 / 3$ \\
& iteration/last iteration) & 1 \\
\hline & group and aligning data & \\
\hline
\end{tabular}

relevant principle component analysis (RPCA $)^{16}$ and partial least squares regression (PLSR) ${ }^{17}$ All programming and statistical analyses were performed using R (R-Project R, http://www.r-project.org/).

The RPCA algorithm is a blind and nonsupervised method by which samples are grouped together, and relevant features, discriminating between the samples in the time course, can be captured. ${ }^{18}$ Time-course fermentation metabolic direction can be observed in relevant PC's scores and interpreted by using the relevant PC's loadings. These indicate the fragments and compounds that are formed and consumed during the fermentation process, as well as measuring the contribution of them in the different fermentations. Chromatograms were normalized by the internal standard (3-octanol) by mean scaling and division by standard deviation for the assignment of the same degree of importance to the preprocessing resulting metabolites. It is important to note that the normalization can be considered an artifact for data interpretation as it is attributed the same degree of importance to the true signal and to noise, and thus, metabolites that are considered to be important to explain samples metabolic profile in each fermentation time can correspond not to valid compounds but to noisy compounds.

The mass spectra features of each volatile compound were validated using two methods: (1) target features by retention time correspondence and (2) correlation between features documented in the NIST 98 MS library.

PLSR was mainly used to determine metabolic coexpression between known and/or unknown compounds present in GC-MS chromatograms. PLSR decomposes the covariance matrix between the sample chromatogram and the supervised metabolites matrix to develop a linear relationship between them, using only the projections that maximize the correlation between the two data sets. Thus, coexpression between supervised metabolites and all metabolites present in a GC-MS chromatogram can be studied by analyzing the PLS coefficients. Therefore, the amount of metabolites obtained by direct peak width integration in the supervised target methodology can be plotted against the overall folded matrix, obtained from the nontarget approach, for classification and validation purposes. In this case, we used the unfold PLSR technique (U-PLSR). ${ }^{19,20}$ The aligned chromatograms form a trilinear tensor that can be unfolded into a single matrix. U-PLSR follows the same algorithms of PLSR, ${ }^{18}$ maximizing the unfold chromatogram covariance matrix, to obtain the U-PLSR. After U-PLSR algorithm application, peaks and fragments highly correlated to the supervised metabolites can be visualized as a chromatogram, where positively correlated peaks are coexpressed with the reference compounds and the negatively correlated peaks are inversely correlated. This allows for the analysis and validation of compounds corresponding to the holistic approach that are being consumed or produced in the fermentation through time. 

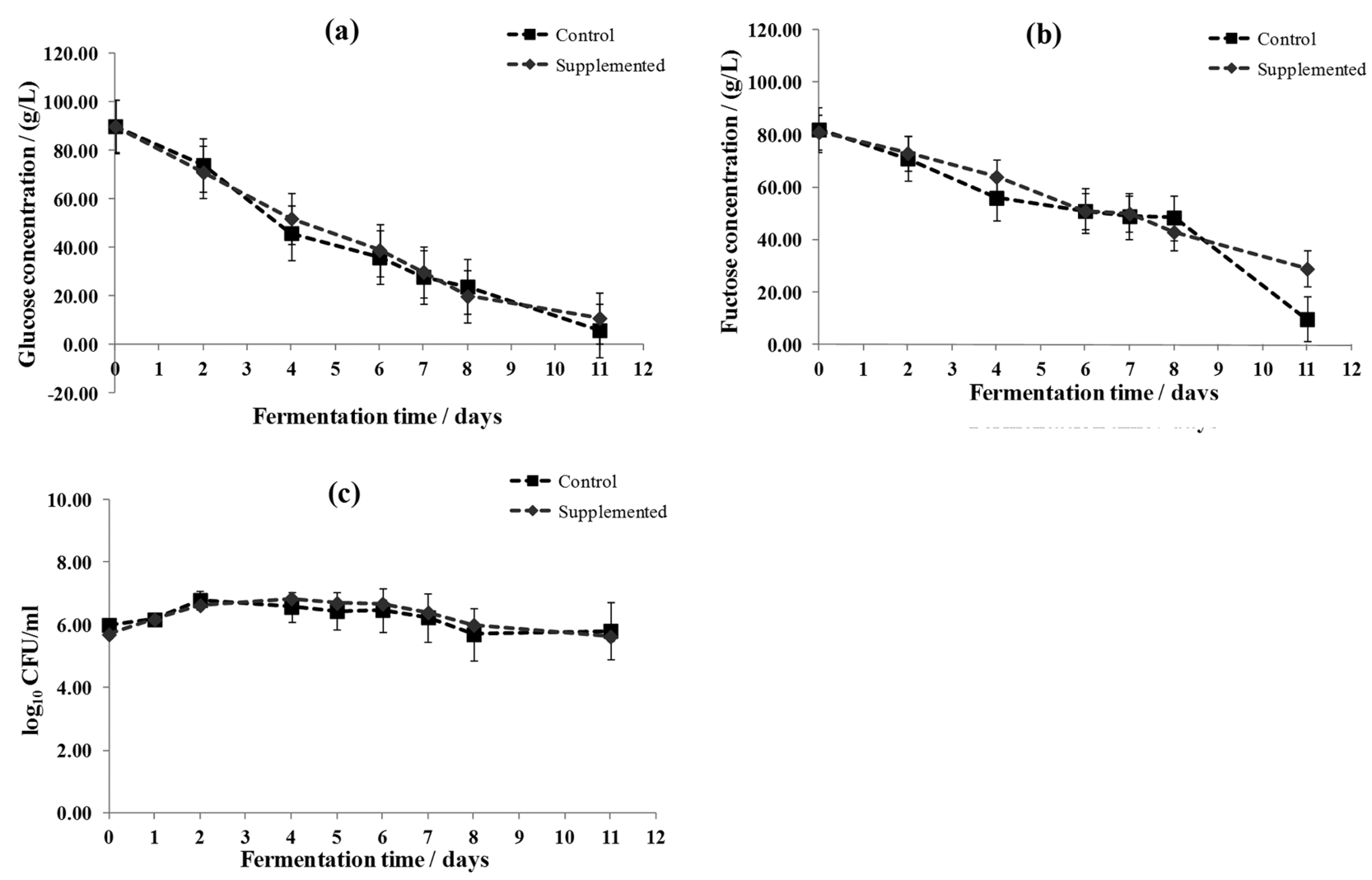

Figure 3. Fermentation monitoring kinetic parameters in both control and supplemented medium: (a) glucose concentrations/(g/L), (b) fructose concentrations $/(\mathrm{g} / \mathrm{L})$, and $(\mathrm{c})$ cell concentrations $/\left(\log _{10} \mathrm{CFU} / \mathrm{mL}\right)$.
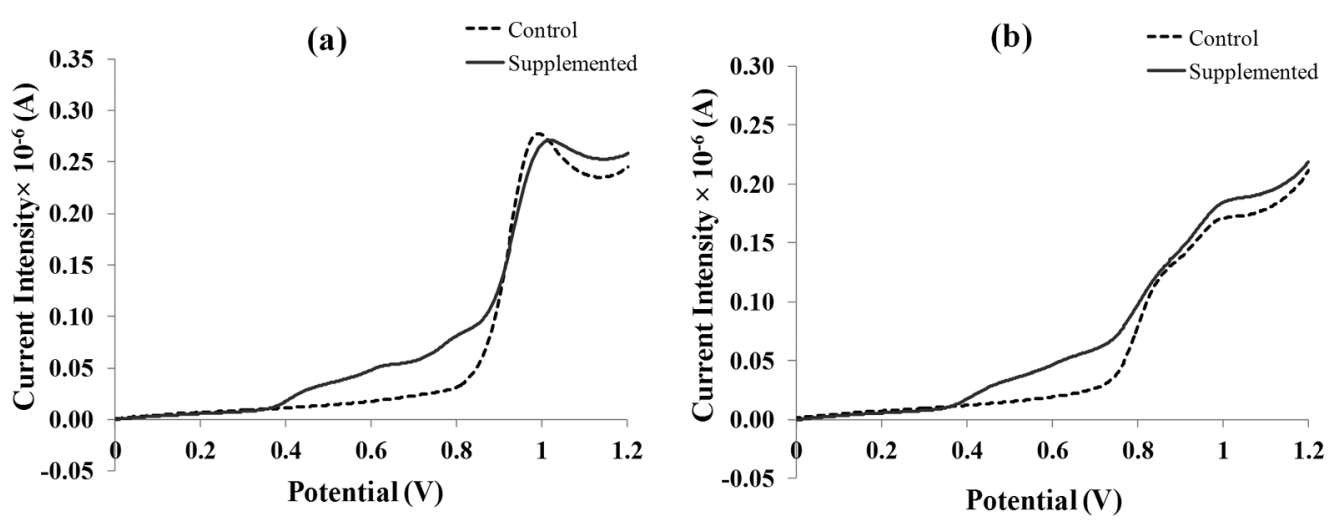

Figure 4. Electrochemical signals of control (dashed line) and supplemented (full line) fermentations in (a) the beginning of the fermentation process $\left(T_{0}\right)$ and $(b)$ the end of the fermentation process $\left(T_{11}\right)$.

A set of compounds, including those identified as the most contributory (presented in the loadings plot) for samples differentiation and dispersion through the RPCA scores plot, as well as other known compounds that participate with those in certain pathways, were identified in a target mode. This was done by comparison with mass spectra, obtained from the samples, with the retention times of pure commercially available standards injected using the same conditions, and by comparing the Kovats indices and the mass spectra present in the NIST 98 MS library. These selected compounds were then normalized to the internal standard, in a selected ion current mode. Ions selected were, respectively, for (IS) 3octanol (99\%, Sigma) $m / z=83$; 2-phenylethanol (98\%, SigmaAldrich) $\mathrm{m} / z=91$; phenylacetaldehyde (90\%, Sigma-Aldrich) $\mathrm{m} / z=$ 91; isoamyl acetate (95\%, Sigma-Aldrich) $m / z=43$; isoamyl alcohol (98\%, Sigma-Aldrich) $m / z=55$; acetic acid (99.5\%, Sigma) $m / z=43$; and 2-methoxy-4-vinylphenol (98\%, Sigma) $m / z=150$. Results from the target approach were used to compare and validate other key compounds obtained in the nontarget approach, and the combination of both approaches allows us to obtain a valid interpretation of the overall process.

\section{RESULTS AND DISCUSSION}

Fermentation Performance. The effect of phenolic acids supplementation in yeast growth and the fermentation performance were studied during alcoholic fermentations using a control (in the absence of phenolic acids) against a supplemented (with phenolic acids) medium (Figure 2). Each fermentation was monitored for 11 days, and the kinetics of its control parameters (fructose, glucose, and viable plate counts) were recorded. Comparing the kinetics of monitoring parameters of both control and supplemented fermentations, no statistically differences were found between them $(p<0.05)$ 

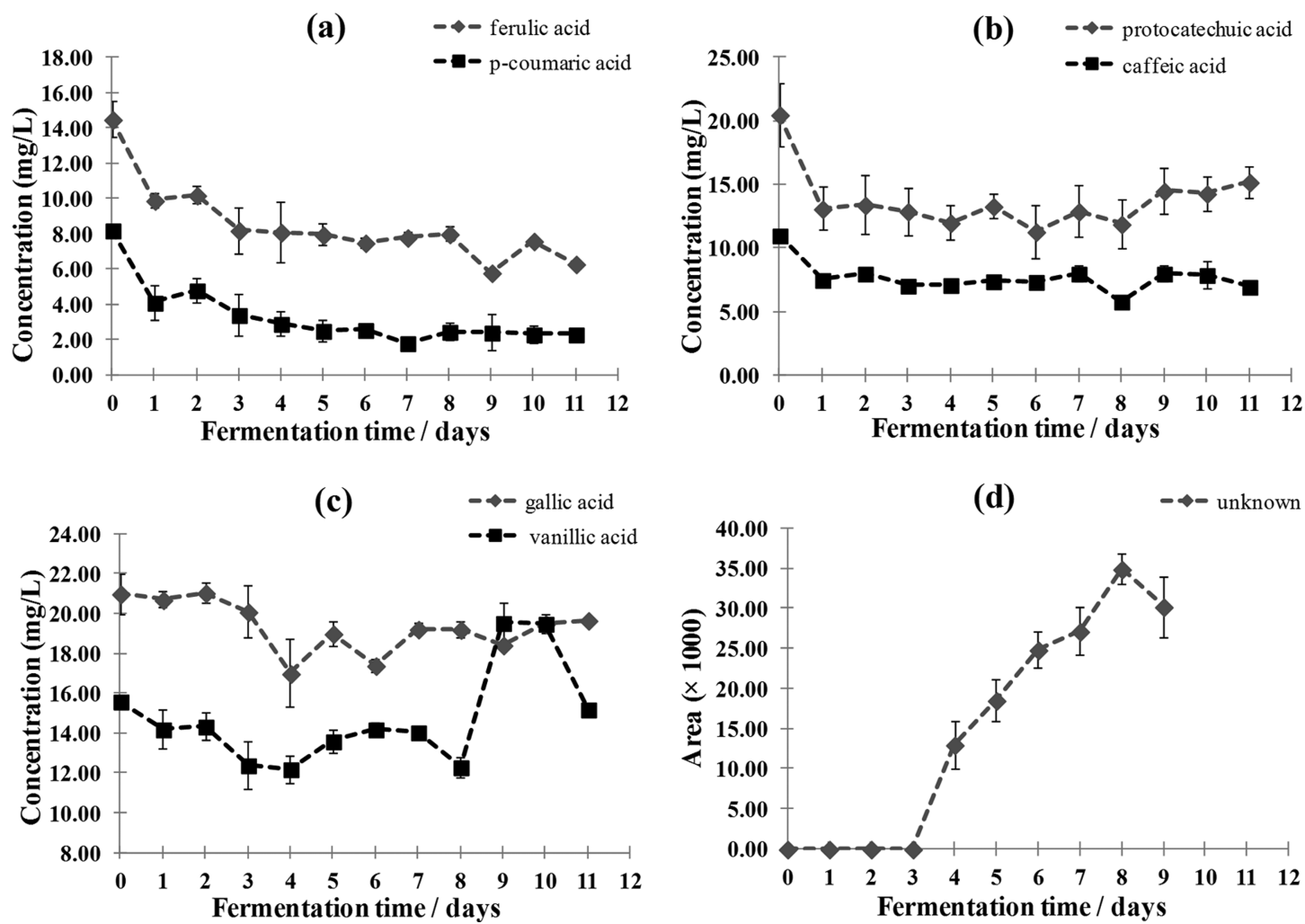

Figure 5. Phenolic acids kinetics during the supplemented fermentation process: (a) para-coumaric and ferulic, (b) caffeic and protocatechuic, (c) vanillic and gallic, and (d) unknown compound.

(Figure 3). The maximum viable cell density of $S$. cerevisiae obtained reached around $10^{7} \mathrm{CFU} / \mathrm{mL}$ from $10^{6} \mathrm{CFU} / \mathrm{mL}$ inocula, as expected in this SGJ fermentations. ${ }^{21}$ Similar viable plate count behavior in the control and supplemented fermentations indicates that the addition of phenolic acids does not inhibit yeast growth and fermentation performance, not affecting fructose or glucose consumption, also.

Electrochemical Analysis. Cyclic voltammetry was used for electroactive activity scanning, providing a holistic vision of the entire fermentation process, which later should be tentatively understood and validated with selected molecules by the HPLC-DAD analysis. The oxidation curves from both control (dashed line) and supplemented medium (full line) are represented in Figure 4, at the beginning, $T_{0}$ (Figure 4a), and at the end, $T_{11}$ (Figure $4 \mathrm{~b}$ ), of the fermentation process. In Figure 4 , it is possible to observe different electrochemical signals between the control and the supplemented media (dashed and full lines, respectively) as well as different profiles in the beginning and end of the fermentation processes (Figure $4 a, b$, respectively) as a result of a higher concentration of electroactive compounds in the beginning of the fermentation process. Supplemented media voltammograms (full line) display peaks at lower potentials $(0.4-0.8 \mathrm{~V})$, which can be reasonably assumed to be due to the added phenolic compounds (as the control voltammogram does not show this deformation in this potential range). Precise identification of these compounds, related to each peak's position, cannot be ascertained; nevertheless, their structure influences the anodic peak position, as it has been previously reported in the literature. ${ }^{7}$ Using the compound's structure and the evidence from published studies, it can be tentatively assumed that in the potential range $(0.4-0.8 \mathrm{~V})$, gallic acid has the lowest formal potential, followed by caffeic and protocatechuic acids, ferulic and vanillic acids, and para-coumaric acid. ${ }^{7}$ The higher number of hydroxyl substituents present on the benzene ring decreases the formal potential of the phenolic compound. ${ }^{22}$ Gallic acid, a benzoic acid derivative with three available hydroxy groups, is therefore easily oxidized. ${ }^{6,23}$ In the same way, caffeic and protocatechuic acids have an easily oxidizable ortho-diphenol group, which makes them more easily oxidizable than a phenolic acid with an isolated phenol group like para-coumaric acid. The other phenolics that have significantly higher formal potentials (ferulic and vanillic acids) lacked an ortho-diphenol, which is dependent on the oxidation of an isolated phenol group adjacent to a methoxy group. These compounds are therefore expected to be less active as antioxidants where reducing ability is the key requirement. The oxidation of these phenolics, which could be due to processes involving one or two electrons, ${ }^{24}$ produced broad peaks and is largely irreversible. ${ }^{6}$ The type of electroactive compounds changed during fermentation ( $T_{0}$ to $\left.T_{11}\right)$, as indicated by the voltammograms (Figure 4a,b). Voltammograms clearly show a decrease in the amplitude of the broad band at approximately $1 \mathrm{~V}$. Conversely, one broad band at $0.8 \mathrm{~V}$ is brought about by fermentation, indicating that a change in the overall resistance to oxidation does indeed occur during alcoholic fermentation and shows a pattern similar to that of normal wines. ${ }^{4}$ It must be remembered that our medium is of a considerably minimal nature and that it is interesting that such a medium produces the same type of electroactive substances as natural must.

HPLC-DAD Analysis. Concurrent with the electrochemical study, the kinetics of the added phenolic acids to the supplemented media were quantified and monitored using HPLC-DAD and are presented in Figure 5. By observing phenolic acids kinetics (Figure 5), it was possible to detect that phenolic acids most affected by fermentation were para- 


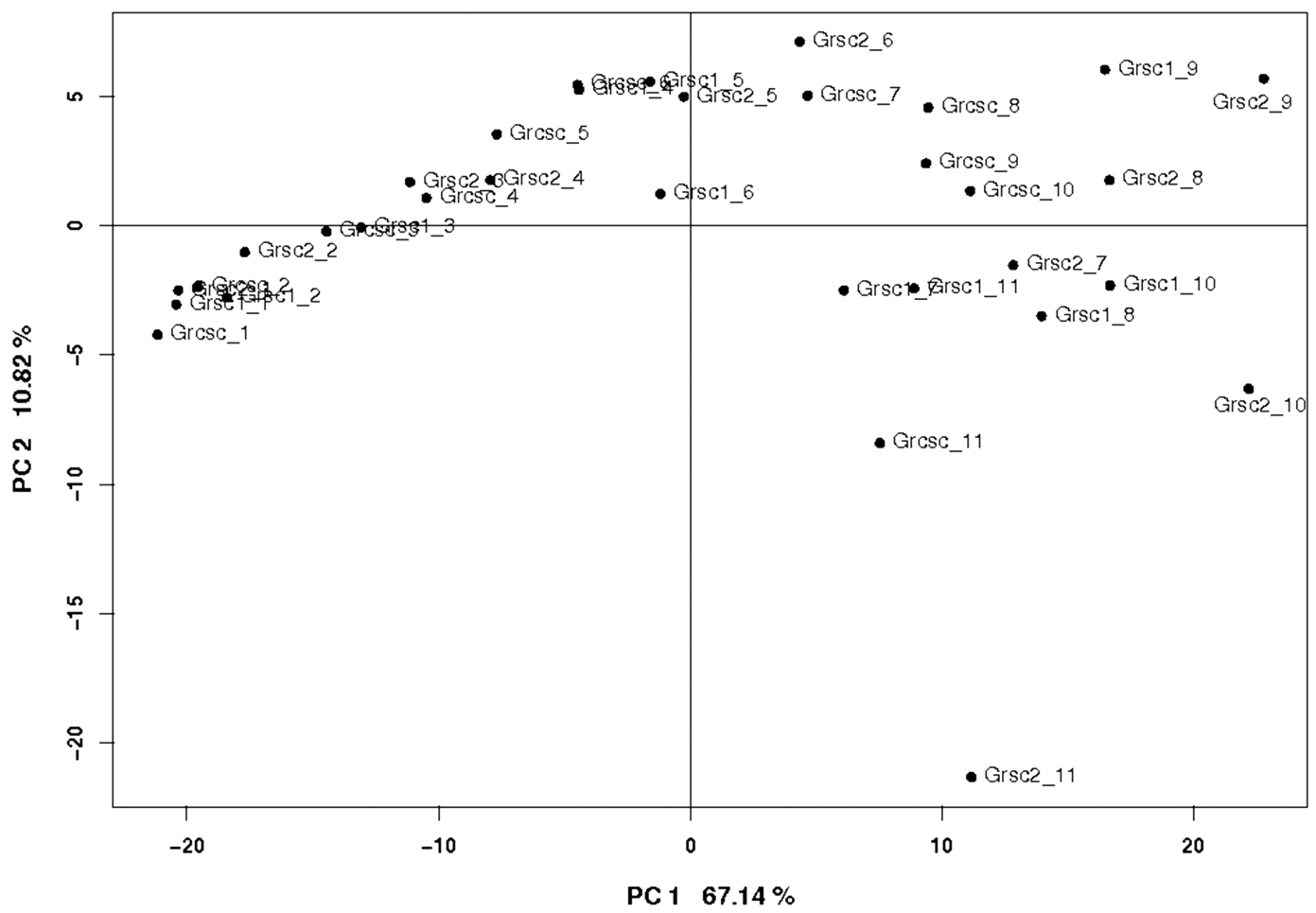

Figure 6. Scores plot resulting from principal component analysis method: PC1 and PC2.

coumaric ( $\square$ ) and ferulic acids $(\boldsymbol{})$ (Figure 5a). In fact, it is known that during the fermentation process, para-coumaric and ferulic acids can be used for 4-vinylphenol and to 2-methoxy-4vinylphenol (4-vinylguaiacol) or vanillic acid formation, respectively. ${ }^{1,25,26}$ In fact, the final concentration of the ferulic acid is lower than the vanillic acid. The phenolic acids presented in Figure 5b,c were not largely changed during the fermentation time course. Protocatechuic acid ( $)$ concentration (Figure $5 b$ ) suffers a small decrease at the beginning of alcoholic fermentation but thereafter remains constant until the end of the time-course sampling time. Caffeic ( $\square$ ) (Figure $5 b)$, gallic $(\boldsymbol{})$, and vanillic ( $\mathbf{\square})$ (Figure 5c) acids did not show any significant change in concentration throughout the fermentation. Besides the study of the added phenolic compounds, one compound of particular interest was found increasing in both control and supplemented media, after 5 days of alcoholic fermentation, whose growth pattern, in control medium, is shown in Figure 5d. Attempts were made to further identify this unknown compound, and it was found to have the same retention time as the standard for chlorogenic acid. These results were confirmed by LC-MS analyses (results not shown). The discovery of chlorogenic acid in the control medium was unexpected as $S$. cerevisiae is not a known producer of any phenolic acids. This is of particular interest, because its presence in the control medium suggests that $S$. cerevisiae has the ability to produce antioxidants de novo from simple precursors such as sugars and amino acids. Chlorogenic acid is the condensation product of quinic and caffeic acids; furthermore, it is a product of an intermediate in aromatic acid biosynthesis and a late intermediate of phenol biosynthesis. ${ }^{27}$ While it is not documented that yeast produces this acid, S. cerevisiae does contain some of the enzymes and certain pathways used by plants for its production. S. cerevisiae performs aromatic acid de novo biosynthesis via the Shikimate pathway, ${ }^{25}$ creating a possible pathway for the production of quinic acid. Furthermore, the exploration of the Saccharomyces Genome Database (SGD) shows that the yeast contains the PAL gene (phenylalanine ammonia lyase, enzyme: 4.3.1.5), which encodes the enzyme needed to catalyze the deamination of phenylalanine to trans-cinnamate and ammonia. These results clearly indicate that more research and more thorough study are necessary to validate and understand the reasons for production of chlorogenic acid during alcoholic fermentation.

Metabolomic Analysis. In addition to the electrochemical changes, it is known that during the fermentation of wine, different biological processes occur, which lead to different organoleptic characteristics of the final product, depending on the conditions of the fermentation medium. ${ }^{1}$ In this context, it seems noticeable that through the interpretation of the holistic view that can be provided by the nontarget analysis of the overall process as well as the kinetics of individual compounds of interest in target mode, it may be possible to understand the metabolic phenomena concomitant with assimilation or processing of phenolic compounds added the fermentation medium.

After the chromatograms preprocessing using the MetAlign software, ${ }^{15}$ the resulting data set was subjected to a nonsupervised approach for classifying the metabolic information. This included the RPCA analysis, which was used to impose a statistical structure on the preprocessed HS-SPME/GC-MS obtained data set. Figure 6 presents the samples scores Gabriel plot, where principal component 1 (PC1) and principal component 2 (PC2) have a discriminated power of 67.14 and $10.82 \%$, respectively, totaling approximately $78 \%$ of the chemical information explanation.

Samples distribution in the PC1 vs PC2 space presented in Figure 6 shows similar features of the control fermentation samples (Grcsc) as compared to the supplemented mediareplicas 1 ( Grsc1) and 2 ( $\mathrm{Grsc} 2)$. PC1 is the most relevant component and is the result of the variable time during the 


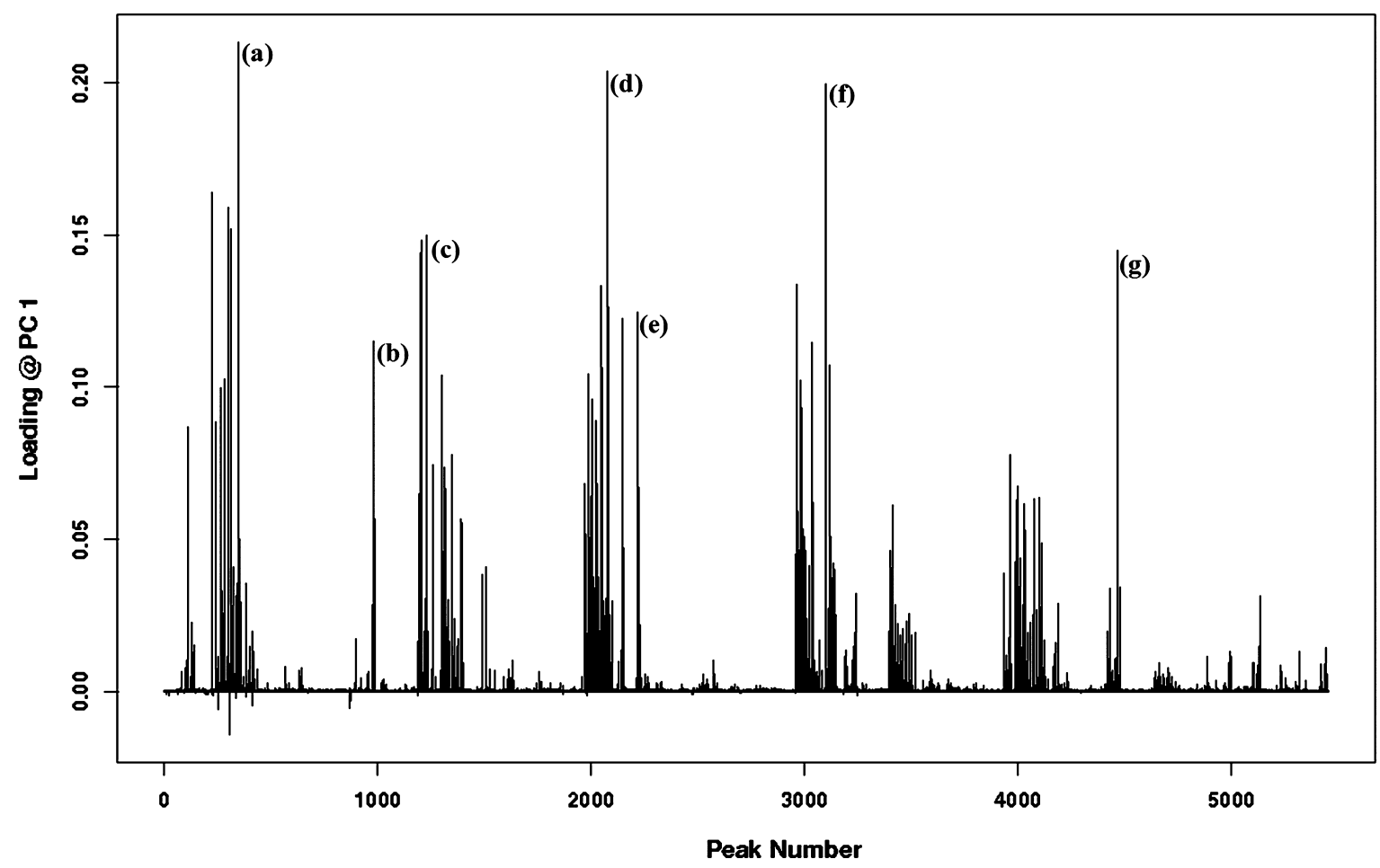

Figure 7. Loadings plot corresponding to the PC1, resulting from principal component analysis method (the annotation of the highlighted fragments is presented in Table 2).

fermentation process, as samples distributed in the PC1 axis space have a time dependence. This demonstrates that the metabolic profile is changing over time, during the fermentation process in all media. The interpretation of the metabolic information that differentiates samples throughout the fermentation can be done analyzing the loadings of the singular value decomposition analysis, represented in Figure 7 and described in Table 2. Peaks reported in Figure 7 correspond to

Table 2. Tentative Identification of the Loadings Plot Fragments Corresponding to the First Principal Component Resulting from the Principal Component Analysis Method

\begin{tabular}{|c|c|c|c|c|}
\hline label & $\begin{array}{l}\text { peak } \\
\text { no. }\end{array}$ & $\begin{array}{c}\text { scan } \\
\text { no. }\end{array}$ & $m / z$ & $\begin{array}{l}\text { tentative } \\
\text { identification }\end{array}$ \\
\hline $\mathrm{a}$ & 349 & 389 & $\begin{array}{l}46(1) ; 73(0.19) ; 92(0.09) \text {; } \\
47(0.05)\end{array}$ & unknown \\
\hline $\mathrm{b}$ & 980 & 724 & $\begin{array}{l}43(1) ; 55(0.54) ; 70(0.45) \\
41(0.24)\end{array}$ & isoamyl acetate \\
\hline c & 1203 & 975 & 39 (1); $41(0.94) ; 70(0.88)$ & unknown \\
\hline d & 2076 & 1661 & $\begin{array}{l}41(1) ; 173(0.82) ; 57(0.68) \\
\quad 56(0.52)\end{array}$ & unknown \\
\hline e & 2220 & 1712 & $\begin{array}{l}43(1) ; 45(0.78) ; 60(0.32) ; \\
61(0.09)\end{array}$ & acetic acid \\
\hline f & 3101 & 2256 & $\begin{array}{l}41(1) ; 201(0.98) ; 60(0.35) \text {; } \\
\quad 44(0.25)\end{array}$ & unknown \\
\hline g & 4468 & 2983 & $91(1) ; 92(0.63) ; 122(0.22)$ & 2-phenylethanol \\
\hline
\end{tabular}

those who must contribute to samples distribution throughout the PC1 axis ( $x$-axis), in this case, were identified as isoamyl acetate, acetic acid, and 2-phenylethanol (Figure 7b,e,g, respectively), whose kinetics can be found in the target approach (Figure 9). Peaks labeled in Figure 7a,c,d,f were tentatively identified as unknown compounds, and more efforts should be done to interpret the results. In fact, the most laborious task linked to the nontarget approach is the identification step, usually by searching the NIST library and by direct comparison after injection of standards. ${ }^{14}$ In this work, as the main objective is to present a tool to provide an enlarged view of the overall system, we did not carry out a detailed identification and quantification of all of the metabolites.

PLSR was also used to determine metabolic correlations between 2-phenylethanol and all peaks obtained in the GC-MS chromatograms. 2-Phenylethanol fermentation kinetics obtained by direct peak-width integration in the target approach was plotted against the data matrix resulting from MetAlign preprocessing in the nontarget approach, for classification purposes. This metabolite was arbitrarily selected, as the main purpose is to show that besides being possible the characterization of the system holistically, the statistical tools used in this pipeline may also be crucial in understanding the correlations, then coexpressions, of interesting metabolites, which can be correlated with the phenolic acids kinetics during the fermentation process.

Figure 8 shows the resulting display of PLSR coefficients of the model performed using 2-phenylethanol amounts, and higher coefficients correspond to the most correlated scans with 2-phenylethanol. The image map color (blue to red scale) of the point determines the magnitude of correlation, being higher for red. In Figure 8, it is possible to observe that 2phenylethanol is strongly correlated with 2-phenylethanol (scan 2983) (Figure 8a), acetic acid (scan 1712) (Figure 8b), 2,3-dihydro-3,5,dihydroxy-6-methyl-4 $(H)$-pyran-4-one (scan 3802) (Figure 8c), and 5-hydroxymethyl-furfural (scan 4292) (Figure $8 \mathrm{~d}$ ), which means that the kinetics of production of each compound is similar during the fermentation process. The assignment of scan numbers to their corresponding compounds was facilitated by the target approach, which identified a set of metabolites associated with alcoholic fermentation, and 

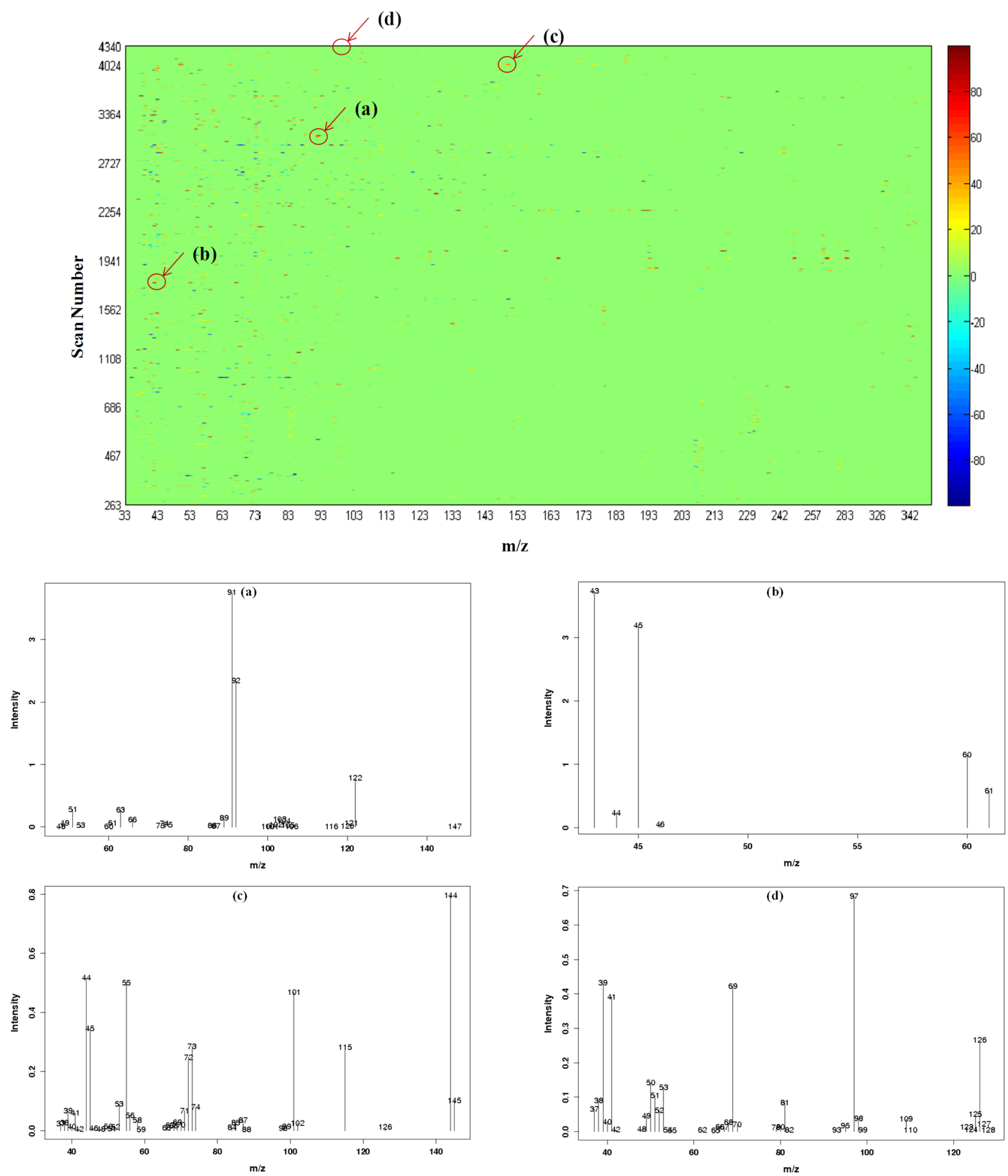

Figure 8. Partial least squares regression resulting coefficients correlations with 2-phenylethanol throughout the fermentation process: (a) 2phenylethanol (scan 2983), (b) acetic acid (scan 1712), (c) 2,3-dihydro-3,5,dihydroxy-6-methyl-4(H)-pyran-4-one (scan 3802), and (d) 5hydroxymethyl-furfural (scan 4292).

validated their mass spectral profiles. Using this classification method and after the identification process, new information about metabolic pathways may be revealed.

The evolution throughout fermentation of some secondary metabolites, including those identified as the most contributory for the explanation of the fermentation progress, is presented in Figure 9. Higher alcohols and esters, including 2-phenylethanol and isoamyl acetate, and those known to be correlated with them (phenylacetaldehyde and isoamyl alcohol), as well as the acetic acid, were identified and quantified during the fermentation process. The fermentation kinetics of each metabolite in both the supplemented and the control media were shown to be statistically similar $(p<0.05)$.

So, in Figure 9a, it is possible to observe a greater increase of the 2-phenylethanol until the eighth day of the process. The production of this fusel alcohol is mainly associated with the 

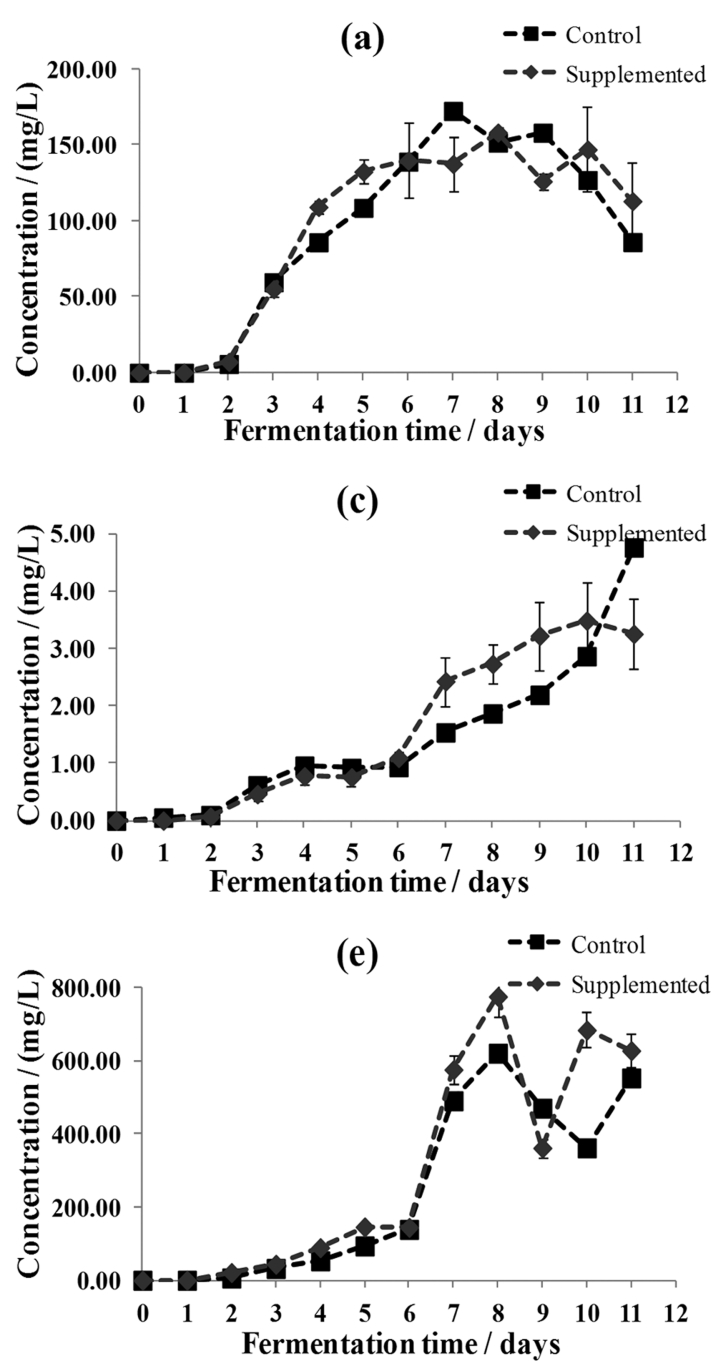

(b)

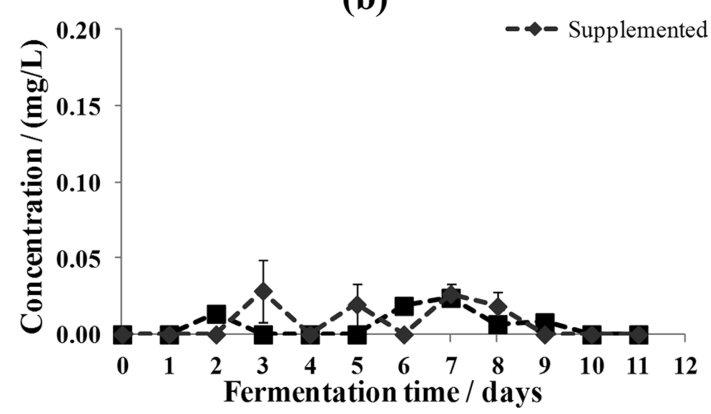

(d)

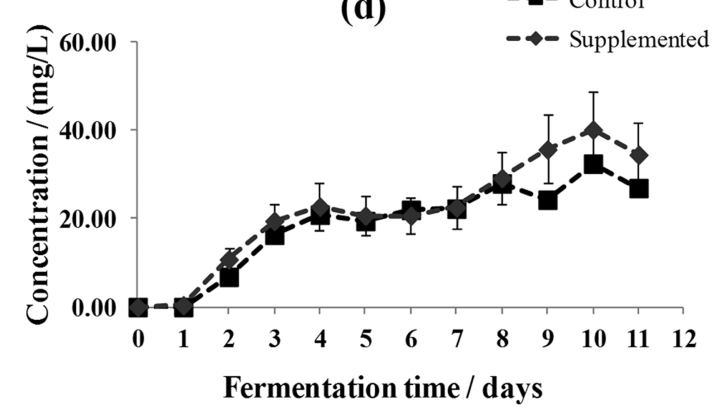

(f)

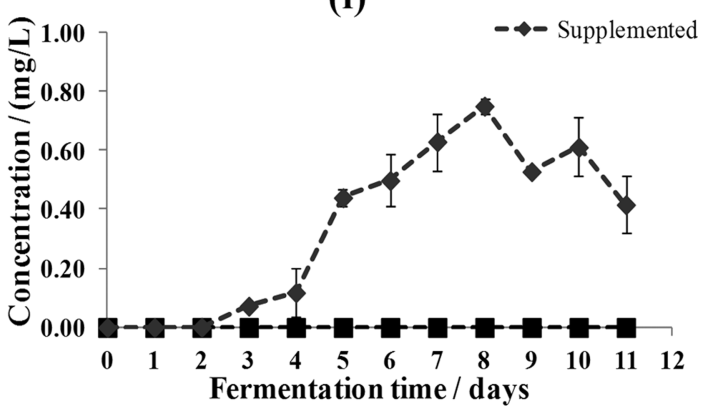

Figure 9. Target analysis of some volatile metabolites kinetics found to be important for samples dispersion in the scores plan and some known to be correlated with those in both control and supplemented medium: (a) 2-phenylethanol, (b) phenylacetaldehyde, (c) isoamyl acetate, (d) isoamyl alcohol, (e) acetic acid, and (f) 2-metoxy-4-vinylphenol.

phenylalanine degradation by the Ehrlich pathway, ${ }^{28,29}$ where phenylacetaldehyde is an intermediary compound (being consumed as is produced, as it is presented in Figure $9 \mathrm{~b})$.

Comparing both 2-phenylethanol and phenylacetaldehyde kinetics, it is possible to confirm their relationship, as when the concentration of phenylacetaldehyde decreases to zero, the concentration of 2-phenylethanol no longer increases. This also suggests that phenylalanine, initially added to the SGJ, was totally converted into 2-phenylethanol during the fermentation processes by $S$. cerevisiae after 8 days of fermentation.

The synthesis of isoamyl acetate (Figure 9c) by S. cerevisiae is catalyzed by a group of enzymes called alcohol acetyltransferase (AAT) by utilizing higher alcohols, in this case the isoamyl alcohol (Figure 9d), and acetyl-CoA (resulting from pyruvate metabolism) as substrates. ${ }^{1}$ The amount of isoamyl acetate and isoamyl alcohol appears to increase throughout the control and supplemented fermentation processes. This means that as isoamyl alcohol is being produced, $S$. cerevisiae provides the conductive and continuous condition for the production of isoamyl acetate. As isoamyl alcohol is the result of L-leucine degradation by the Ehrlich pathway, ${ }^{28,29}$ it is also suggested that this amino acid is present in both fermentation media throughout the fermentation processes.
Acetic acid is also a normal end product of the alcoholic fermentation, ${ }^{1,30}$ and its concentration seems to increase until the eighth day of both fermentation processes, decreasing from then onward until the end of fermentation (Figure 9e). In this case, as glucose is constantly consumed (Figure 3a) for the formation of the end products (i.e., ethanol, glycerol, and acetic acid), the final decrease of the acetic acid amount could be due to a residual substrate concentration available. Comparing 2methoxy-4-vinylphenol kinetics in both the supplemented and the control fermentations (Figure 9f), it is possible to verify different behaviors between them. In the supplemented media, the amount of this volatile phenol also increases until the eighth day; however, in the control fermentation, there was no evidence of 2-methoxy-4-vinylphenol production. These kinetic differences in control and supplemented fermentations lead us to suggest that the addition of phenolic acids significantly affected the primary metabolism or rate of production of secondary metabolites. As it was discussed, 2-methoxy-4vinylphenol is the result of ferulic acid metabolism, one of the added phenolic acids. ${ }^{1,25}$

So, the combination of information from different detectors, including cyclic voltammetry and liquid and gas chromatography in this work, revealed exciting prospects to explore and 
gather the maximum information regarding complex systems, such as real must fermentation.

\section{AUTHOR INFORMATION}

\section{Corresponding Author}

*Tel: +351-225580001. Fax: +351-225580001. E-mail: asferreira@porto.ucp.pt / antoniof@sun.ac.za.

\section{Funding}

C.C.C. (SFRH/BD/46737/2008) gratefully acknowledges the Fundação para a Ciência e Tecnologia (FCT) for her Doctoral grant. This research was funded by the projects: "OpenMicrobio (PTDC/BIO/69310/2006)-Framework for Computational Simulation of Cellular Communities during BioProcess Engineering” (FCT), "A Métrica do Vinho: Elucidar o Padrão Molecular Volatile Responsável pelo aroma "Tipo-Vinho" Fundamental para a Definição da Qualidade" (PTDC/AGRALI/121062/2010), and partially supported by CBMA, IBB, and ESB/UCP plurianual funds through the POS-Conhecimento Program that includes FEDER funds.

\section{Notes}

The authors declare no competing financial interest.

\section{REFERENCES}

(1) Swiegers, J. H.; Pretorius, I. S. Yeast modulation of wine flavor. Adv. Appl. Microbiol. 2005, 57, 131-175.

(2) Nicoli, M. C.; Toniolo, R.; Anese, M. Relationship between redox potential and chain-breaking activity of model systems and foods. Food Chem. 2004, 88 (1), 79-83.

(3) Leistner, L.; Gorris, L. G. M. Food preservation by hurdle technology. Trends Food Sci. Technol. 1995, 6 (2), 41-46.

(4) Rodrigues, A.; Silva Ferreira, A. C.; Guedes de Pinho, P.; Bento, F.; Geraldo, D. Resistance to oxidation of white wines assessed by voltammetric means. J. Agric. Food Chem. 2007, 55 (26), 1055710562

(5) Waterhouse, A. L.; Laurie, V. F. Oxidation of wine phenolics: A critical evaluation and hypotheses. Am. J. Enol. Vitic. 2006, 57 (3), 306-313.

(6) Kilmartin, P. A.; Zou, H.; Waterhouse, A. L. A cyclic voltammetry method suitable for characterizing antioxidant properties of wine and wine phenolics. J. Agric. Food Chem. 2001, 49 (4), 1957-1965.

(7) Fiehn, O. Metabolomics-The link between genotypes and phenotypes. Plant Mol. Biol. 2002, 48 (1), 155-171.

(8) Rodrigues, J. A.; Barros, A. S.; Carvalho, B.; Brandão, T.; Gil, A. M.; Ferreira, A. C. S. Evaluation of beer deterioration by gas chromatography-mass spectrometry/multivariate analysis: A rapid tool for assessing beer composition. J. Chromatogr., A 2011, 1218 (7), 990996.

(9) Tikunov, Y.; Lommen, A.; de Vos, C. H.; Verhoeven, H. A.; Bino, R. J.; Hall, R. D.; Bovy, A. G. A novel approach for nontargeted data analysis for metabolomics. Large-scale profiling of tomato fruit volatiles. Plant Physiol. 2005, 139 (3), 1125-1137.

(10) Weckwerth, W.; Morgenthal, K. Metabolomics: From pattern recognition to biological interpretation. Drug Discovery Today 2005, 10 (22), 1551-1558.

(11) Ciani, M.; Ferraro, L. Enhanced Glycerol Content in Wines Made with Immobilized Candida stellata Cells. Appl. Environ. Microbiol. 1996, 62 (1), 128-132.

(12) Jackson, R. Wine Science: Principles and Applications, 3rd ed.; Academic Press: San Diego, 2008.

(13) Demiray, S.; Pintado, M. E.; Castro, P. M. L. Evaluation of phenolic profiles and antioxidant activities of Turkish medicinal plants: Tilia argentea, Crataegi folium leaves and Polygonum bistorta roots. World Acad. Sci., Eng. Technol. 2009, 54, 312-317.

(14) Silva Ferreira, A. C.; Hogg, T.; Guedes de Pinho, P. Identification of key odorants related to the typical aroma of oxidation-spoiled white wines. J. Agric. Food Chem. 2003, 51 (5), 1377-81.

(15) Lommen, A. MetAlign: Interface-driven, versatile metabolomics tool for hyphenated full-scan mass spectrometry data preprocessing. Anal. Chem. 2009, 81 (8), 3079-86.

(16) Martins, R. C.; Lopes, V. V.; Valentão, P.; Carvalho, J.; Isabel, P.; Amaral, M. T.; Batista, M. T.; Andrade, P. B.; Silva, B. M. Relevant principal component analysis applied to the characterisation of Portuguese heather honey. Nat. Prod. Res. 2008, 22 (17), 1560-1582.

(17) Abdi, H. Partial Least Squares Regression (PLS-Regression); Sage: Thousand Oaks, CA, 2003; pp 792-795.

(18) Huang, J.; Wium, H.; Qvist, K. B.; Esbensen, K. H. Multi-way methods in image analysis-relationships and applications. Chemom. Intell. Lab. Syst. 2003, 66 (2), 141-158.

(19) Kiers, H. A. L. Towards a standardized notation and terminology in multiway analysis. J. Chemom. 2000, 14 (3), 105-122.

(20) Chevion, S.; Roberts, M. A.; Chevion, M. The use of cyclic voltammetry for the evaluation of antioxidant capacity. Bio-assays Oxidative Stress Status (BOSS) 2001, 120.

(21) Péres-Nevado, F.; Albergaria, H.; Hogg, T.; Gírio, F. Cellular death of two non-Saccharomyces wine-related yeasts during mixed fermentations with Saccharomyces cerevisiae. Int. J. Food Microbiol. 2006, 108 (3), 336-345.

(22) Rice-Evans, C. A.; Miller, N. J.; Paganga, G. Structureantioxidant activity relationships of flavonoids and phenolic acids. Free Radical Biol. Med. 1996, 20 (7), 933-956.

(23) Hendrickson, H. P.; Sahafayen, M.; Bell, M. A.; Kaufman, A. D.; Hadwiger, M. E.; Lunte, C. E. Relationship of flavonoid oxidation potential and effect on rat hepatic microsomal metabolism of benzene and phenol. J. Pharm. Biomed. Anal. 1994, 12 (3), 335-341.

(24) Shinohara, T.; Kubodera, S.; Yanagida, F. Distribution of phenolic yeasts and production of phenolic off-flavors in wine fermentation. J. Biosci. Bioeng. 2000, 90 (1), 90-97.

(25) Herrmann, K. M.; Weaver, L. M. The shikimate pathway. Annu. Rev. Plant Biol. 1999, 50 (1), 473-503.

(26) Mathew, S.; Abraham, T. E.; Sudheesh, S. Rapid conversion of ferulic acid to 4-vinyl guaiacol and vanillin metabolites by Debaryomyces hansenii. J. Mol. Catal. B: Enzym. 2007, 44 (2), 48-52.

(27) Braus, G. H. Aromatic amino acid biosynthesis in the yeast Saccharomyces cerevisiae: A model system for the regulation of a eukaryotic biosynthetic pathway. Microbiol. Mol. Biol. Rev. 1991, 55 (3), 349.

(28) Ehrlich, F. Über die Bedingungen der Fuselölbildung und über ihren Zusammenhang mit dem Eiweissaufbau der Hefe. Ber. Dtsch. Chemischen Gesellschaft 1907, 40 (1), 1027-1047.

(29) Hazelwood, L. A.; Daran, J. M.; van Maris, A. J.; Pronk, J. T.; Dickinson, J. R. The Ehrlich pathway for fusel alcohol production: A century of research on Saccharomyces cerevisiae metabolism. Appl. Environ. Microbiol. 2008, 74 (8), 2259-66.

(30) Ludovico, P.; Sousa, M. J.; Silva, M. T.; Leão, C.; Corte-Real, M. Saccharomyces cerevisiae commits to a programmed cell death process in response to acetic acid. Microbiology 2001, 147 (9), 2409. 